\title{
Application of Harmonic Balance-Finite Element Method (HBFEM) in the Design of Switching Power Supplies
}

\author{
Jun Wei Lu, Member, IEEE, Sotoshi Yamada, Member, IEEE, and H. Barry Harrison
}

\begin{abstract}
Numerical modeling and analysis are effective techniques to assist in the design of high-frequency switching power supplies. Striving for high power densities and higher efficiencies has pushed the operating frequencies up, resulting in new circuit configurations and different component architecture. This paper presents a numerical method that is used to analyze magnetic systems for switching power supplies, in particular the switching resonant dc-dc converters. The method uses the harmonic balance technique combined with finite element methods and is referred to as the harmonic balance-finite element method (HBFEM). The HBFEM is used to solve various harmonic, eddy-current, hysteresis, and nonlinear problems applied to magnetic circuits used in switching power supplies. To evaluate the numerical results, comparisons are made with experimental results.
\end{abstract}

\section{INTRODUCTION}

$\mathbf{N}$ UMERICAL modeling techniques are becoming popular for electromagnetic field analysis of high-frequency, high-density switching power supplies. Other numerical techniques for the solution of structural stress and thermal analysis are also finding favor with the design engineer. This paper discusses mainly the numerical modeling and analysis of electromagnetic fields in switching power supplies. The advantages of such power supplies are high efficiency, small size, and lower weight [1]. These advantages do not come free of charge because electromagnetic interference (EMI), leakage inductance, skin and proximity effects, winding selfcapacitance, and interwinding capacitance can present serious problems. Furthermore, the nonlinear nature and hysteresis of the core material can cause waveform distortion [2], [3]. These waveform distortions cause harmonics that will increase power losses in both the winding and magnetic core and cause a loss of efficiency as well as the possibility of parasitic resonance in the system unless properly designed.

Here we use harmonic balance analysis combined with the finite element method to solve problems arising from nonlinear, harmonic, eddy-current, and power loss problems of transformers used in switching power supplies.

\section{The Concept of Harmonic Balance ANALYSIS APPLIED TO MAGNETIC CIRCUITS}

Harmonic balance techniques have been used to solve

Manuscript received December 7, 1993; revised August 11, 1995

The authors are with the School of Microelectronic Engineering, Faculty. of Science and Technology, Griffith University, QLD 4111, Australia.

Publisher Item Identifier S 0885-8993(96)01924-2. nonlinear circuits problems since the 1960's. This method was firmly established in the 1970's and widely used in solving nonlinear circuits problems and analyzing harmonic problems in the 1980's [4]. It was introduced to quasi-static electromagnetic field analysis at the end of the 1980's [5].

The harmonic balance-finite element method (HBFEM) differs from traditional finite-element time-domain methods, transient analysis, and other time harmonic methods. The harmonic balance uses a linear combination of sinusoids to generate a solution and represents waveforms using the coefficients of the sinusoids. It is combined with the finite element method to solve time-periodic steady-state nonlinear electromagnetic field problems. The HBFEM directly solves the steady-state response of the electromagnetic field in the frequency domain and so is often considerably more efficient than traditional time-domain methods when fields exhibit widely separated time constants and mildly nonlinear behavior.

The electromagnetic field with harmonics satisfies Maxwell's equations. The magnetic core of a converter that has nonlinear characteristics and hysteresis and is excited by a current source of current density $J_{s}$, is considered. From Maxwell's equations, the potential equation of the magnetic field can be written

$$
\Delta \times \nu \Delta \times A+\sigma(\partial A / \partial t+\Delta \varphi)-J_{s}=0
$$

where $\nu(=1 / \mu)$ and $\sigma$ are magnetic reluctivity and conductivity. The vector potential $A$, scalar potential $\varphi$, and current density $J_{s}$ can be, respectively, expressed using trigonometric functions as

$$
\begin{aligned}
A^{i} & =A_{0}^{i}+\sum_{k=1}^{\infty}\left\{A_{k s}^{i} \sin (k \omega t)+A_{k c}^{i} \cos (k \omega t)\right\} \\
\varphi^{i} & =\varphi_{0}^{i}+\sum_{k=1}^{\infty}\left\{\varphi_{k s}^{i} \sin (k \omega t)+\varphi_{k c}^{i} \cos (k \omega t)\right\} \\
J_{s} & =J_{0}+\sum_{k=1}^{\infty}\left\{J_{k s} \sin (k \omega t)+J_{k c} \cos (k \omega t)\right\}
\end{aligned}
$$

where $k s$ and $k c$ indicate the $k$ th $\sin$ and cos coefficients, respectively, and $i$ is the node number of the element in the analyzed spatial domain.

If the nonlinear material properties are isotropic, and variable with magnetic fields, the response of nonlinear materials can also be expressed by assuming a trigonometric function. 


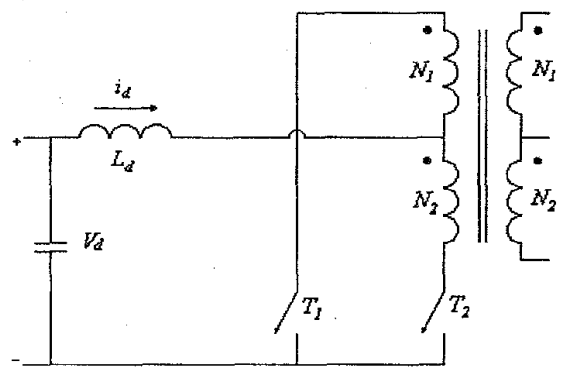

(a)

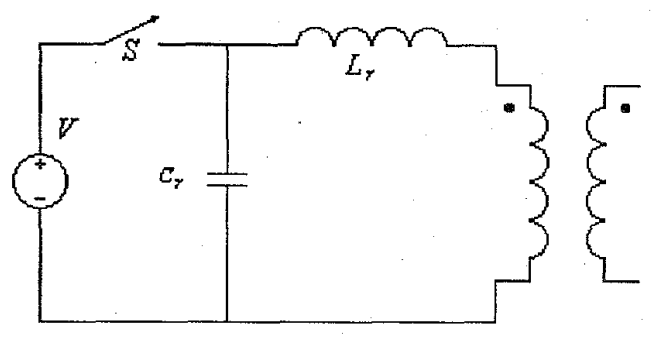

(b)

Fig. 1. Current-source excitation to magnetic field. (a) Switch-mode push-pull converter. (b) Zero-voltage switched resonant converter.

The magnetic reluctivity can be expressed as

$$
\begin{aligned}
\nu(B(t)) & =H\{B(t)\} / B(t) \\
& =\nu_{0}+\sum_{k=2 n-2}^{\infty}\left\{\nu_{k s} \sin (k \omega t)+\nu_{k c} \cos (k \omega t)\right\}
\end{aligned}
$$

where the Fourier coefficients can be calculated from

$$
\begin{aligned}
\nu_{0} & =\frac{1}{T} \int_{0}^{T} \nu(t) d t \\
\nu_{n s} & =\frac{2}{T} \int_{0}^{T} \nu(t) \cdot \sin (n \omega t) d t \\
\nu_{n c} & =\frac{2}{T} \int_{0}^{T} \nu(t) \cdot \cos (n \omega t) d t .
\end{aligned}
$$

If the hysteresis characteristic of the magnetic core is considered, the approximate expression can be used to describe magnetization and residual hysteresis [6], that is

$$
\begin{aligned}
H\{B(t)\} & =H_{0}(B)+H_{e} \frac{d B}{d t} \\
& \approx\left(\alpha B+\beta B^{2 n-1}\right)+\left(\frac{1}{f} \frac{d B}{d t}\right) \frac{d B}{d t}
\end{aligned}
$$

where the first term indicates the saturation characteristic of the magnetic core and the second term expresses the hysteresis characteristic. $\alpha$ and $\beta$, which are coefficients of saturation, can be obtained from the $B-H$ curve of the magnetic core, and $f$ is the excitation frequency.

Because the trigonometric function has an orthogonal characteristic, the harmonic potential $A_{k}$ on each node of a boundary also satisfies Dirichlet and Neumann boundary conditions, which are the frequency domain representations, and are, respectively, expressed as

$$
\begin{aligned}
A_{k} & =\left\{A_{0}, A_{1 s}, A_{1 c}, A_{2 s}, A_{2 c}, \cdots\right\}^{T} \\
\frac{\partial A_{k}}{\partial n} & =\left\{\frac{\partial A_{0}}{\partial n}, \frac{\partial A_{1 s}}{\partial n}, \frac{\partial A_{1 c}}{\partial n}, \frac{\partial A_{2 s}}{\partial n}, \frac{\partial A_{2 c}}{\partial n}, \cdots\right\}^{T} .
\end{aligned}
$$

Equations (10) and (11) indicate that the magnetic potential $A$ at a boundary is also joined by all harmonic components.

\section{HBFEM with CURRENT Source to MAGNetic FIELD}

In switching power supplies, some dc/dc converter topologies like zero voltage switching resonant converters and pushpull current source converters can be considered as currentsource to magnetic field systems, as shown in Fig. 1. The
HBFEM numerical modeling and computation results compared with experimental results are discussed in this section.

\section{A. Numerical Model of Current Source to Magnetic Field}

Assuming $\Delta \varphi=0$ in the two dimensional case and using Galerkin's method [7] to discretize the governing equation, (1) for the two dimensional problems can be written as

$$
\begin{aligned}
G= & \iint_{S}\left\{\frac{\partial N_{i}}{\partial x} \nu \frac{\partial A}{\partial x}+\frac{\partial N_{i}}{\partial y} \nu \frac{\partial A}{\partial y}\right\} d x d y \\
& -\iint_{S}\left\{J_{s}-\sigma \frac{\partial A}{\partial t}\right\} N_{i} d x d y=0 .
\end{aligned}
$$

Considering the orthogonal characteristic of trigonometric functions, substituting magnetic vector $A$, interpolation functions $N_{i}$, magnetic reluctivity $\nu$, and current density $J_{s}$ into (12), and using harmonic balance techniques, the following harmonic balance matrix equation considering only odd harmonics for a single element can be obtained

$$
\begin{aligned}
G^{e}= & \frac{1}{4 \Delta^{e}} \\
& \times\left[\begin{array}{lll}
\left(b_{1} b_{1}+c_{1} c_{1}\right) D & \left(b_{1} b_{2}+c_{1} c_{2}\right) D & \left(b_{1} b_{3}+c_{1} c_{3}\right) D \\
\left(b_{2} b_{1}+c_{2} c_{1}\right) D & \left(b_{2} b_{2}+c_{2} c_{2}\right) D & \left(b_{2} b_{3}+c_{2} c_{3}\right) D \\
\left(b_{3} b_{1}+c_{3} c_{1}\right) D & \left(b_{3} b_{2}+c_{3} c_{2}\right) D & \left(b_{3} b_{3}+c_{3} c_{3}\right) D
\end{array}\right] \\
& \times\left\{\begin{array}{l}
A_{1}^{e} \\
A_{2}^{e} \\
A_{3}^{e}
\end{array}\right\}+\frac{\sigma \omega \Delta^{e}}{12}\left[\begin{array}{ccc}
2 N & N & N \\
N & 2 N & N \\
N & N & 2 N
\end{array}\right]\left\{\begin{array}{l}
A_{1}^{e} \\
A_{2}^{e} \\
A_{3}^{e}
\end{array}\right\} \\
& -\left\{\begin{array}{l}
K_{1}^{e} \\
K_{2}^{e} \\
K_{3}^{e}
\end{array}\right\}=\left[S^{e}\right]\left\{A^{e}\right\}+\left[N^{e}\right]\left[A^{e}\right]-\left\{K^{e}\right\}
\end{aligned}
$$

where the vector potential $\left\{A^{e}\right\}$ is called the frequencydomain representation, $\Delta$ is the area of the triangular elements, and $\omega$ indicates the fundamental frequency. The $b$ and $c$ are obtained from $x$ and $y$ coordinates $\left(b_{i e}=x_{j e} y_{k e}-\right.$ $\left.x_{k e} y_{j e}, c_{i e}=y_{j e}-y_{k e}\right)$. The $D$ and $N$ matrix blocks are

$D=\left[\begin{array}{ccccc}d_{11} & d_{12} & d_{13} & d_{14} & \cdots \\ d_{21} & d_{22} & d_{23} & d_{24} & \cdots \\ d_{31} & d_{32} & d_{33} & d_{34} & \cdots \\ d_{41} & d_{42} & d_{43} & d_{44} & \cdots \\ \cdots & \cdots & \ldots & \ldots & \ddots\end{array}\right]$ 


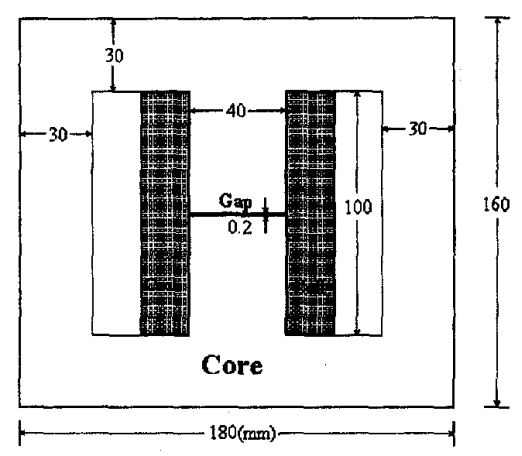

(a)

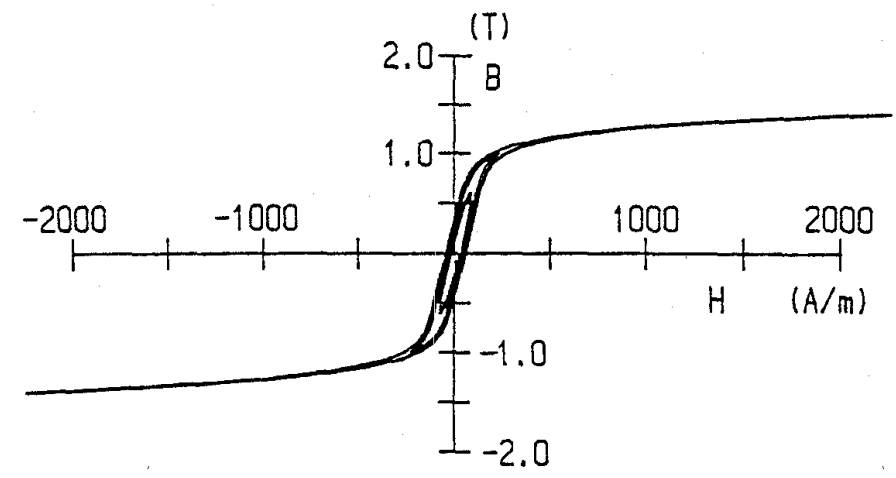

(b)

Fig. 2. Magnetic system with current-source excitation. (a) Magnetic configuration. (b) Hysteresis characteristic.

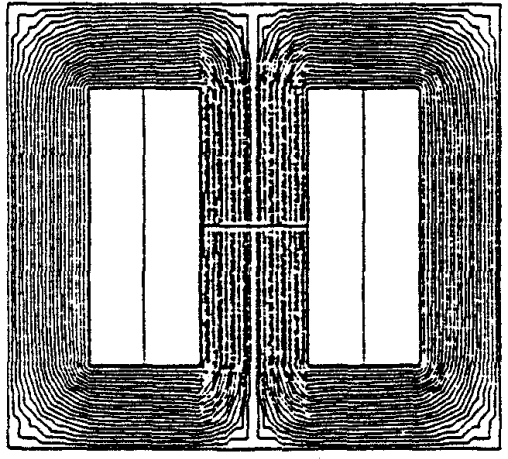

(a)

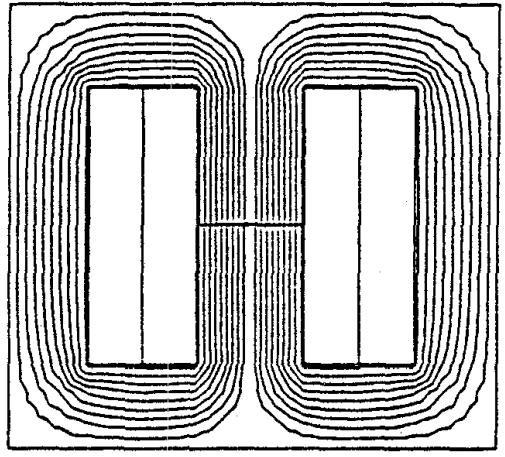

(b)

Fig. 3. The magnetic flux distribution for fundamental and third harmonic components. (a) Fundamental component: $\omega t=90$. (b) Third harmonic component: $3 \omega t=90$.

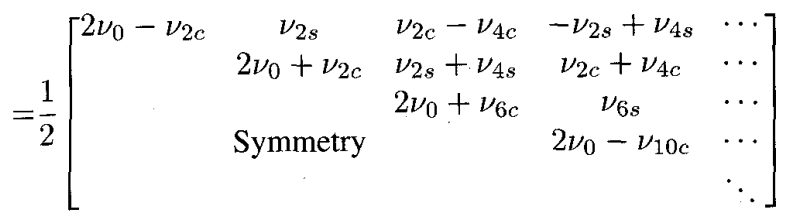

and

$$
N=\left[\begin{array}{rrrrrrr}
0 & -1 & 0 & 0 & 0 & 0 & \cdots \\
1 & 0 & 0 & 0 & 0 & 0 & \cdots \\
0 & 0 & 0 & -3 & 0 & 0 & \cdots \\
0 & 0 & 3 & 0 & 0 & 0 & \cdots \\
0 & 0 & 0 & 0 & 0 & -5 & \cdots \\
0 & 0 & 0 & 0 & 5 & 0 & \ldots \\
\cdots & \cdots & \cdots & \cdots & \cdots & \ldots & \ddots
\end{array}\right] .
$$

Finally, $\left\{K_{i}^{e}\right\}$ is expressed as

$$
\left\{K_{i}^{e}\right\}=\frac{\Delta^{e}}{3}\left\{\begin{array}{lllllll}
J_{1 s} & J_{1 c} & J_{3 s} & J_{3 c} & \ldots, & \cdots, & \cdots
\end{array}\right\}^{T} .
$$

The system matrix equation for current source excitation can be then written as

$$
[S]\{A\}+[M]\{A\}-\{K\}=0 .
$$

All the harmonic components of $A$ can be obtained by solving this matrix equation.

\section{B. Example of Current-Source Excitation to Nonlinear Magnetic Field}

The magnetic components of power supplies are usually excited by time-periodic quasi-static signals, and therefore the magnetic field can be considered as a quasi-static field. If the excitation is a sinusoidal wave, due to the magnetic saturation, harmonics occur in the magnetic field and current. These harmonics will increase power losses and EMI. This example shows the numerical analysis of nonlinear magnetic fields for power supplies.

In zero-voltage switched (ZVS) resonant converters and switching mode push-pull converters inserting an inductor at the input side, the excitation can be considered as a currentsource to the magnetic field. The configuration of the magnetic system is shown in Fig. 2, where the hysteresis characteristic is considered as shown in Fig. 2(b). If the magnetic core is excited by a sinusoidal wave, a harmonic flux will occur in the magnetic field, as shown in Fig. 3, which is calculated by HBFEM [8], [9].

To validate the numerical results, the analysis model with an air gap and two slots at the central leg, as shown in Fig. 4, has been used. This structure significantly affects the magnetic 


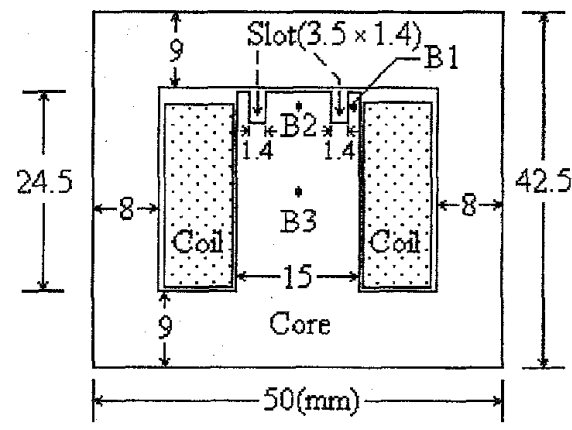

(a)

\section{( $T$ )}

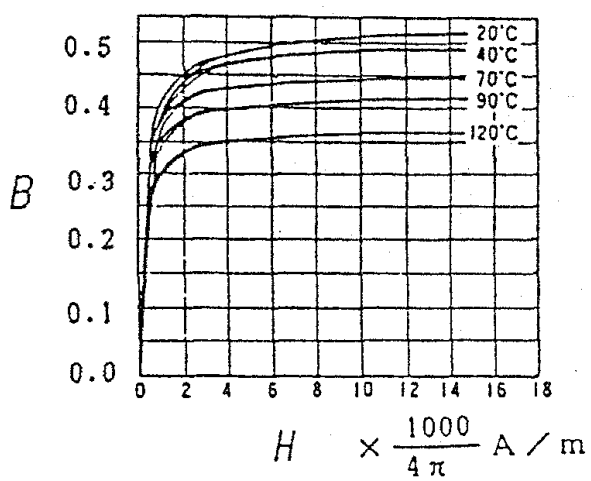

(b)

Fig. 4. Magnetic system with current-source excitation. (a) Magnetic configuration: gap length $=0.19$, depth $=15$, number of turns $=225$. (b) $B-H$ curve.

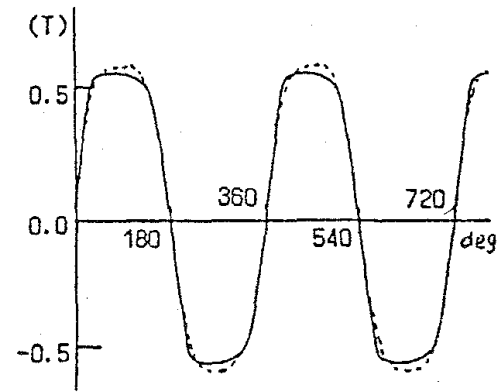

(a)

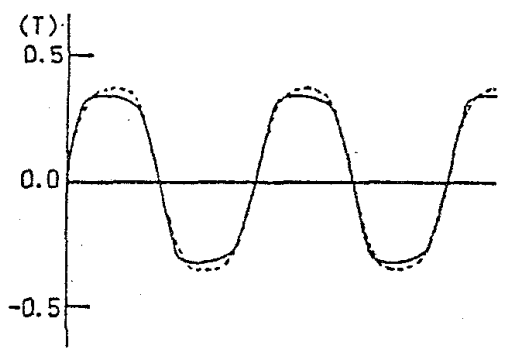

(c)

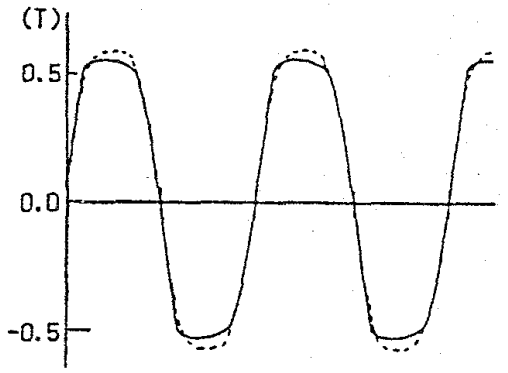

(b)

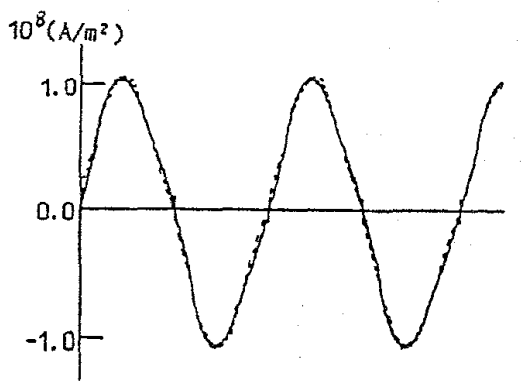

(d)

Fig. 5. The experimental results compared with numerical computation results in the case of current source excitation, where $\cdots$ indicates an experimental result and - indicates a numerical result. (a) Magnetic density $B 1$. (b) Magnetic density $B 2$. (c) Magnetic density $B 3$. (d) Excitation current density $J$.

flux distribution and density in the central leg. The numerical results in Fig. 5 demonstrate that the magnetic density at three different positions has good agreement with experimental results in the case of current source excitation. The computed results also present the magnetic flux distribution for fundamental and third harmonic components as shown in Fig. 6.

\section{HBFEM With VOLTAGE SOURCE TO MAGNETIC SYSTEM}

In most cases, pulse width modulation and zero-current switched resonant converters can be considered as a voltagesource to the magnetic system, which is always coupled to the external circuits, as shown in Fig. 7. The current in the input circuits will be unknown, but saturation of the current waveform occurs because of the nonlinear characteristic of the magnetic core. This section discusses the numerical model and computed results compared with experimental results.

\section{A. Numerical Model of Voltage Source to Magnetic System}

According to Faraday's and Kirchhoff's laws, the relationship between the voltage and the magnetic field for a single element can be found as

$$
\left\{V_{k}\right\}=\left[C_{k}\right]\left\{\begin{array}{l}
\left\{A^{1}\right\} \\
\left\{A^{2}\right\} \\
\left\{A^{3}\right\}
\end{array}\right\}
$$



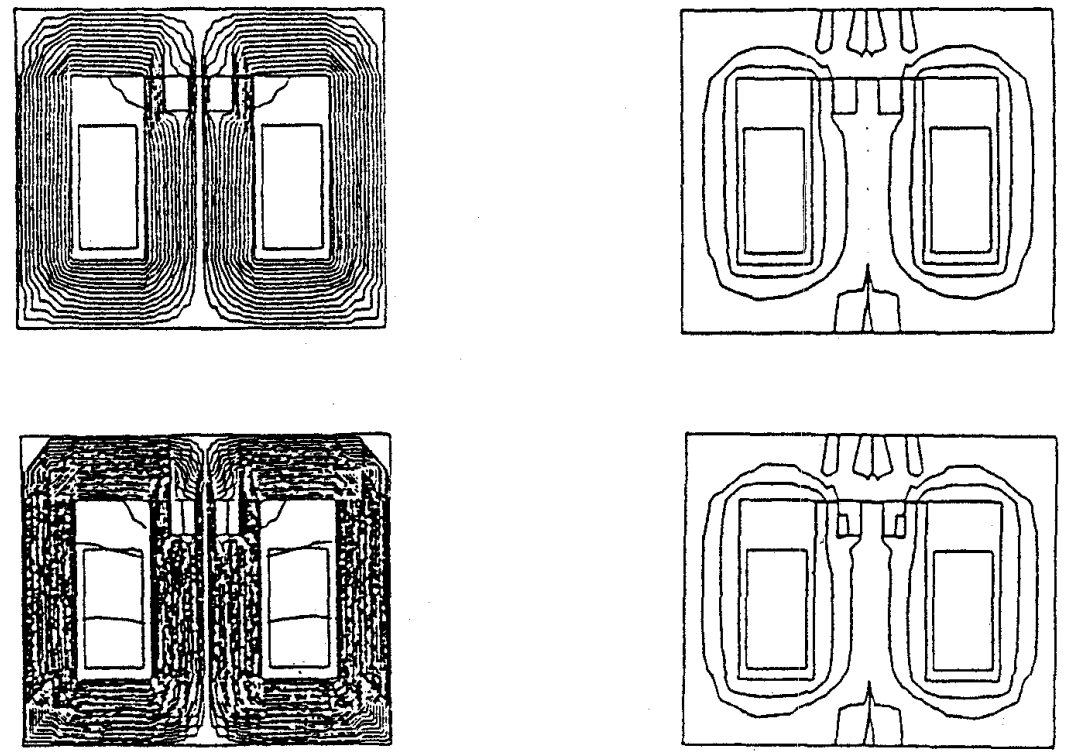

(a)

(b)

Fig. 6. Distribution of magnetic flux in the magnetic core. (a) Fundamental component: above, $\omega t=60$; below, $\omega t=90$. (b) Third harmonic component: above, $3 \omega t=60$; below, $3 \omega t=90$.

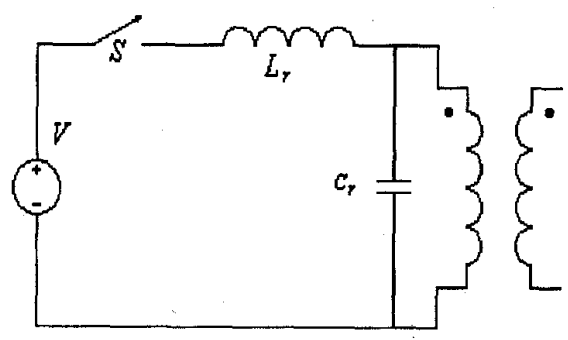

(a)

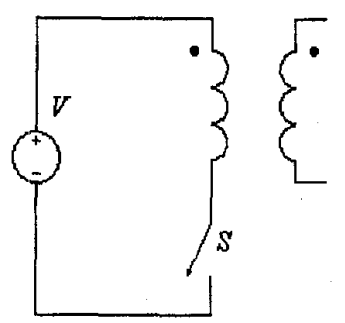

(b)

Fig. 7. Magnetic system coupled to the voltage sources. (a) Zero-current switched resonant converter. (b) Switching mode forward converter.

where matrix $\left[C_{k}\right]$ is the coefficients related to the voltage

$$
\left[C_{k}\right]=\frac{\omega d_{0} \Delta^{e}}{3 S_{c k}}\left[\begin{array}{lll}
N & N & N
\end{array}\right]
$$

$S_{c k}$ and $d_{0}$ are the area of windings and the depth in the $z$-direction, respectively. Then the input voltage can be defined

$$
\left\{V_{\mathrm{in} k}\right\}=\left\{V_{k}\right\}+S_{c k}\left[Z_{k}\right]\left\{J_{k}\right\}
$$

where matrix $\left[Z_{k}\right]$ is the circuit impedance including the resistance of windings corresponding to the harmonics, that is

$$
\left[Z_{k}\right]=\left[\begin{array}{ccc}
{\left[Z_{0 k}\right]} & 0 & 0 \\
0 & {\left[Z_{1 k}\right]} & 0 \\
0 & 0 & \ddots
\end{array}\right]
$$

The $\left[Z_{n k}\right]$ in $(21)$ can be expressed as

$$
\left[Z_{n k}\right]=\left[Z_{R n k}\right]+\left[Z_{C n k}\right]+\left[Z_{L n k}\right]
$$

for the series circuit connection. The input voltage $\left\{V_{\mathrm{ink}}\right\}$ including all the harmonic components that have a known value are expressed as

$$
\left\{V_{\text {ink }}\right\}=\left\{V_{0 k}, V_{1 s k}, V_{1 c k}, V_{2 s k}, V_{2 c k}, \cdots\right\}^{T} .
$$

The system matrix equation related to current can be rewritten as

$$
[S]\{A\}+[M]\{A\}-\left[G_{k}\right]\left\{J_{k}\right\}=0
$$

where $\left[G_{k}\right]$ is obtained from a single element, that is

$$
\left[G^{e}\right]=\frac{\Delta^{e}}{3} .
$$

Combining (20) and (24), the global system matrix equations for multiple input and output are obtained. The harmonic balance FEM matrix equation for voltage source excitation, therefore can be expressed as

$$
\left[\begin{array}{cccccc}
{[H]} & -\left[G_{1}\right] & -\left[G_{2}\right] & \cdots & -\left[G_{k}\right] & \cdots \\
{\left[C_{1}\right]} & S_{c 1}\left[Z_{1}\right] & 0 & 0 & 0 & \cdots \\
{\left[C_{2}\right]} & 0 & S_{c 2}\left[Z_{2}\right] & 0 & 0 & \cdots \\
\vdots & 0 & 0 & \ddots & 0 & \cdots \\
{\left[C_{k}\right]} & 0 & 0 & 0 & S_{c k}\left[Z_{k}\right] & \cdots \\
\vdots & \vdots & \vdots & \vdots & \vdots & \ddots
\end{array}\right]
$$




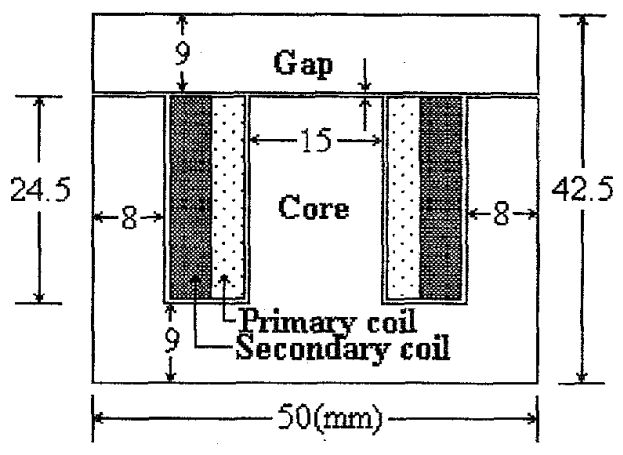

(a)

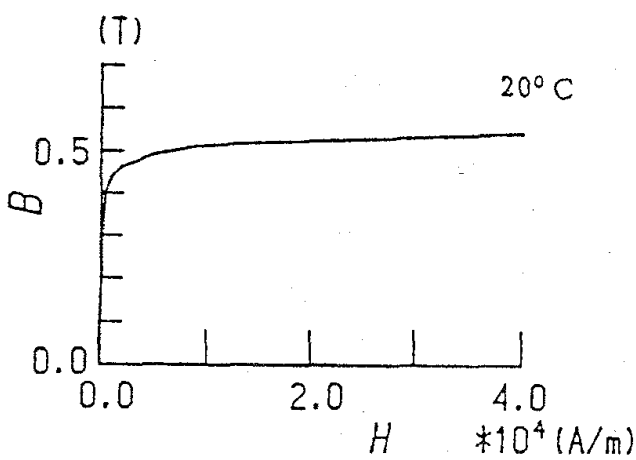

(b)

Fig. 8. Configuration of numerical model for the voltage excitation. 120. (b) Magnetizing characteristics.

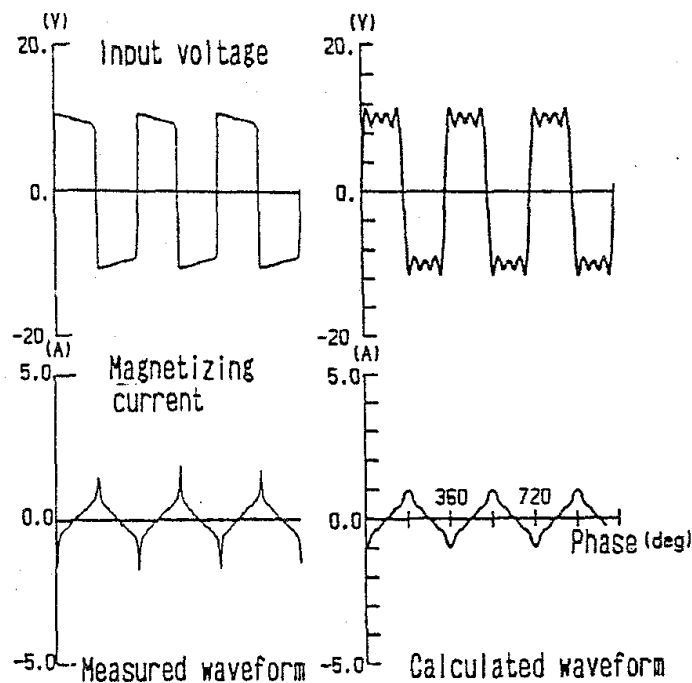

(a)

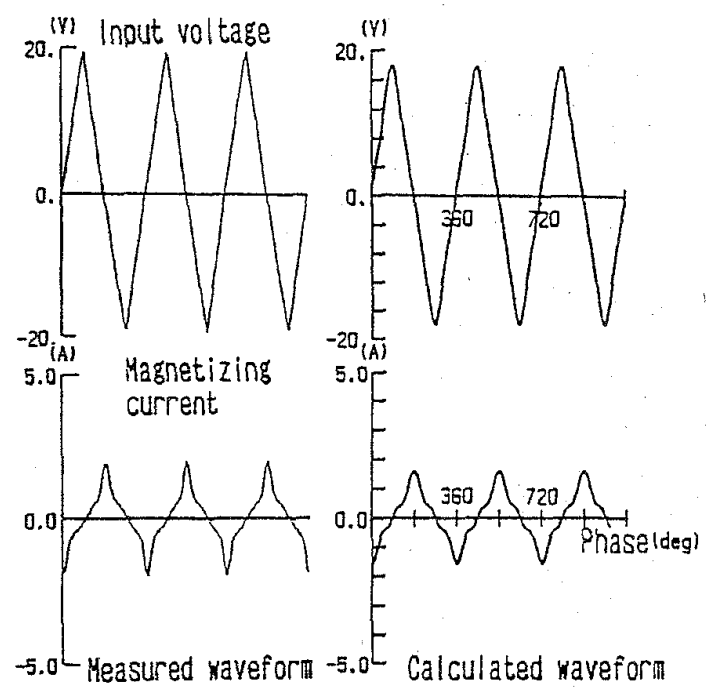

(b)

Fig. 9. Numerical and experimental results for different input voltages. (a) Square wave excitation. (b) Triangular wave excitation.

$$
\times\left\{\begin{array}{c}
\{A\} \\
\left\{J_{1}\right\} \\
\left\{J_{2}\right\} \\
\vdots \\
\left\{J_{k}\right\} \\
\vdots
\end{array}\right\}=\left\{\begin{array}{c}
0 \\
\left\{V_{\text {in } 1}\right\} \\
\left\{V_{\text {in } 2}\right\} \\
\vdots \\
\left\{V_{\text {ink }}\right\} \\
\vdots
\end{array}\right\}
$$

where $\{A\}$ and $\left\{J_{k}\right\}$ are unknown and can be calculated by solving the system matrix equation. $[H]$ is the matrix obtained from $([S]+[M])$.

\section{B. Example of Voltage-Source Excitation to Nonlinear Magnetic Field}

When the transformers of power supplies are excited by a voltage source, such as pulse-width modulation (PWM) converters and zero-current switched (ZCS) resonant converters, the numerical analysis of the magnetic field should be carried out by taking account of the voltage source and the external circuits. If the excitation waveform is a square wave or triangular wave, it can be considered as a linear combination of harmonics. Fig. 8 shows the numerical model of voltage excitation with a nonlinear magnetic field. Fig. 9 demonstrates the numerical analysis results with square and triangular excitations. The distribution of magnetic flux for triangular wave excitation is shown in Fig. 10. The harmonic components of current and magnetic flux can help to more accurately analyze and design the magnetic core and winding structures and determine the power loss and leakage flux in the magnetic system.

\section{CALCUlation OF EDDY CURRENT IN THE WINDINGS}

The eddy current in the transformer and inductor windings has to be kept to the minimum possible because it produces power losses, thereby increasing the thermal requirements and decreasing efficiency. To prevent this kind of undesirable effect in transformers, a good knowledge of the eddy-current distri- 


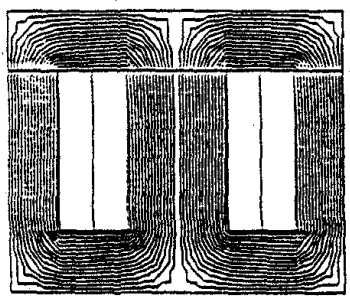

(a)

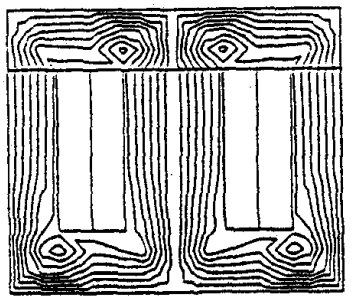

(b)

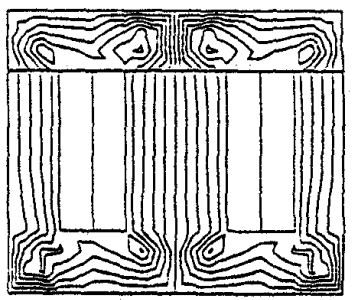

(c)

Fig. 10. Distribution of magnetic flux for triangular wave excitation. (a) Fundamental component. (b) Third harmonic component. (c) Fifth harmonic.

butions and related phenomena is required. The eddy-current distributions can be calculated by using HBFEM, which is more accurate than conventional methods. The numerical model and computed results compared with experimental results are discussed in this section.

\section{A. Numerical Model of Eddy-Current Problems}

The system equations for the eddy-current problem take account of scalar potential $\varphi$ and the dc component is obtained from (1); that is

$$
[S]\{A\}+([M]+[R])\{A\}-\{K\}=0
$$

where $[S]$ and $[M]$ have the same form as in (14), but $D$ and $N$ have different details when the dc component is considered. That is

$$
\begin{aligned}
D & =\left[\begin{array}{ccccc}
d_{11} & d_{12} & d_{13} & d_{14} & \cdots \\
d_{21} & d_{22} & d_{23} & d_{24} & \cdots \\
d_{31} & d_{32} & d_{33} & d_{34} & \cdots \\
d_{41} & d_{42} & d_{43} & d_{44} & \cdots \\
\ldots & \cdots & \cdots & \cdots & \ddots
\end{array}\right] \\
& =\frac{1}{2}\left[\begin{array}{cccccc}
2 \nu_{0} & \nu_{1 s} & \nu_{1 c} & \nu_{2 s} & \cdots \\
2 \nu_{1 s} & 2 \nu_{0}-\nu_{2 c} & \nu_{2 s} & \nu_{1 c}-\nu_{3 c} & \cdots \\
2 \nu_{1 c} & & 2 \nu_{0}+\nu_{2 c} & \nu_{1 s}+\nu_{3 s} & \cdots \\
2 \nu_{2 s} & \text { Symmetry } & & 2 \nu_{0}+\nu_{4 c} & \cdots \\
\vdots & \vdots & \vdots & \vdots & \ddots
\end{array}\right]
\end{aligned}
$$

and

$$
N=\left[\begin{array}{rrrrrrrr}
0 & 0 & 0 & 0 & 0 & 0 & 0 & \cdots \\
0 & 0 & -1 & 0 & 0 & 0 & 0 & \cdots \\
0 & 1 & 0 & 0 & 0 & 0 & 0 & \ldots \\
0 & 0 & 0 & 0 & -2 & 0 & 0 & \cdots \\
0 & 0 & 0 & 2 & 0 & 0 & 0 & \cdots \\
0 & 0 & 0 & 0 & 0 & 0 & -3 & \ldots \\
0 & 0 & 0 & 0 & 0 & 3 & 0 & \ldots \\
\vdots & \vdots & \vdots & \vdots & \vdots & \vdots & \vdots & \ddots
\end{array}\right]
$$

and the eddy-current matrix $\{R\}$ for a single element can be calculated as

$$
\left[R_{i}^{e}\right]=\frac{\left(\Delta^{e}\right)^{2} n \omega \sigma}{12 S}
$$

where $S$ is the cross section of the coil and $\Delta$ is the area of the element.

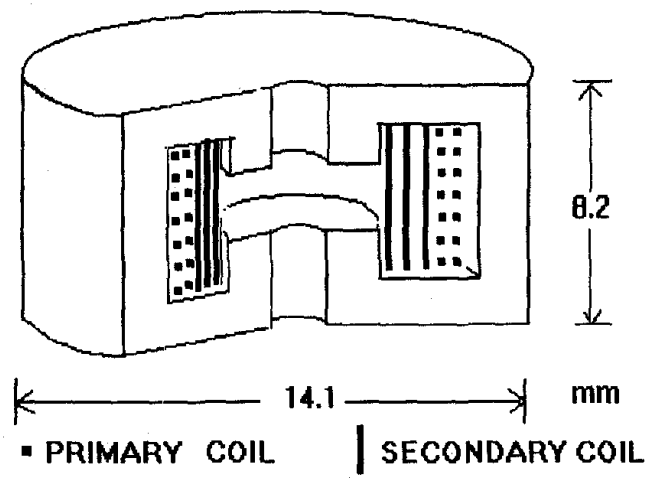

Fig. 11. A typical structure of magnetic core and windings.

\section{B. Numerical Analysis of Eddy Current in the Two-Dimensional Axi-symmetrical Model}

A typical structure of transformers used for resonant switch converters is shown in Fig. 11. In resonant switch converters, the transformer is normally excited by quasi-sinusoidal waveform, which includes ac fundamental, harmonics, and dc components. When magnetic core becomes saturated, the waveforms will be distorted and harmonics will be generated in the magnetic field and circuits. The hysteresis loss is also increased due to the effect of high frequency harmonics. The eddy currents in transformer windings are significantly increased by leakage flux of ac components, especially around the air gap of a magnetic core. Fig. 12 shows the numerical results for the distributions of magnetic flux calculated by HBFEM. From these numerical results, one can find that, around the air gap, the dc component of magnetic flux can easily cross the windings without inducing any current in the windings, while the ac component of magnetic flux is pushed out of the windings by induced current called eddy current in the windings. This eddy current will produce power loss and heating in the windings, which decreases the efficiency. The eddy currents induced in the windings are shown in Fig. 13. The largest current density occurs at the edge of the air gap in the magnetic core. Fig. 14 demonstrates a comparison of numerical and experimental magnetic flux densities.

The power losses produced by eddy currents in both the coil and the magnetic core can be easily calculated by using the numerical results of HBFEM. The formula for the total eddycurrent power loss in the areas of the coil and the magnetic 


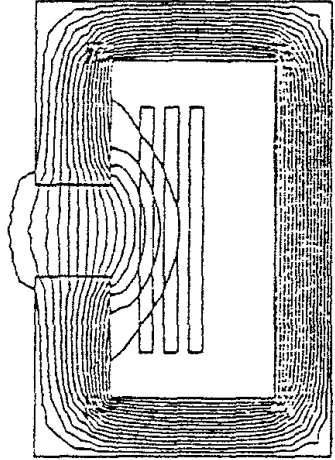

(a)

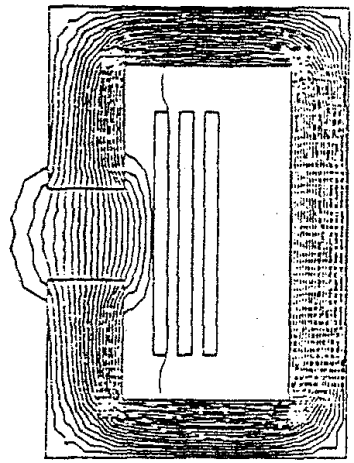

(b)

Fig. 12. Distributions of magnetic fluxes. (a) DC component. (b) Fundamental component.

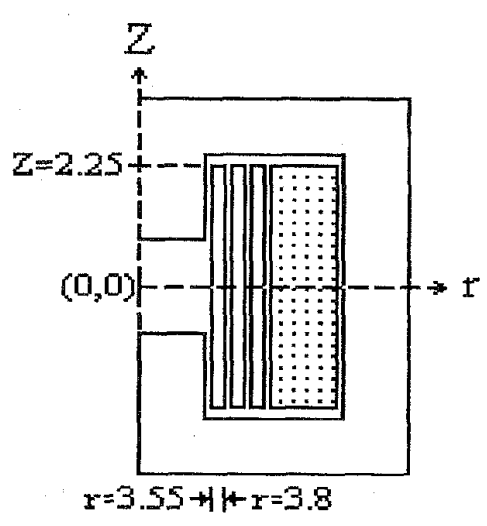

(a)

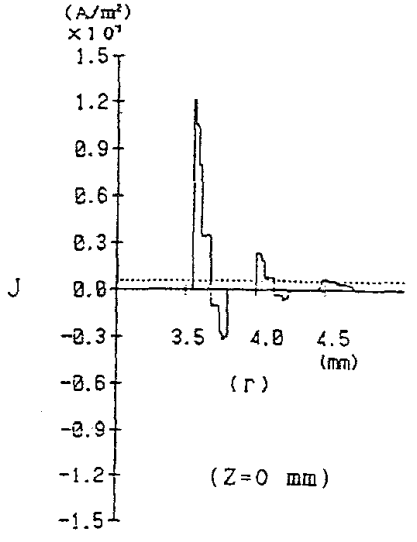

(b)

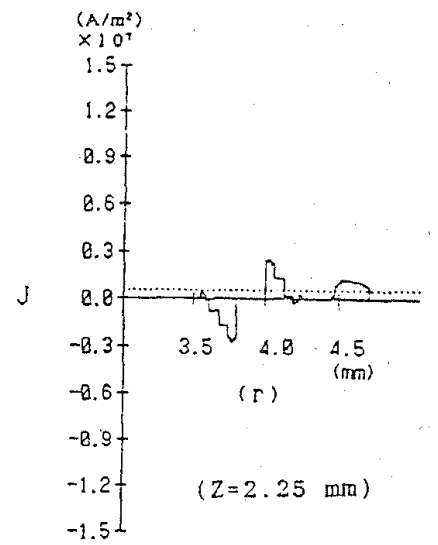

(c)

Fig. 13. Distributions of eddy current in the windings. (a) Axi-symmetrical winding configuration. (b) Eddy current at $Z=0 \mathrm{~mm}$. (c) Eddy current at $Z=2.25 \mathrm{~mm}$.

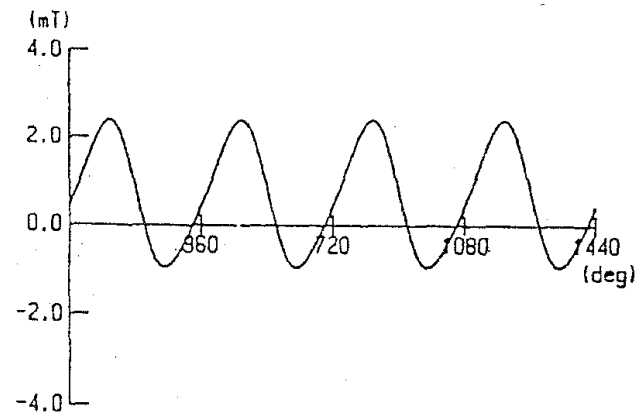

(a)

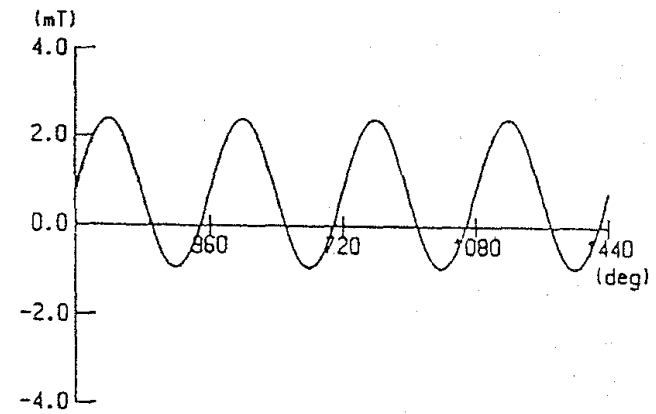

(b)

Fig. 14. Waveforms of magnetic flux density. (a) Experimental result. (b) Numerical result.

core can be expressed as

$$
P_{\text {total }}=P_{\text {coil }}+P_{\text {core }}
$$

where $P_{\text {coil }}$ is the total eddy-current power loss in the coil, given by

$$
P_{\text {coil }}=\sum_{e}^{\text {coil }}\left(P_{1 \text { coil }}^{e}+P_{2 \text { coil }}^{e}+\cdots P_{n \text { coil }}^{e}\right)
$$

and $P_{\text {core }}$ is a summation of the eddy-current power loss in the magnetic core, that is

$$
P_{\text {core }}=\sum_{e}^{\text {core }}\left(P_{1 \text { core }}^{e}+P_{2 \text { core }}^{e}+\cdots P_{n \text { core }}^{e}\right)
$$

where $n$ indicates the $n$th harmonic. Eddy-current power loss for each harmonic component can be calculated using the 
following generalized equation

$$
P_{n}^{e}=\sigma \Delta^{e}\left\{\sqrt{\left(J_{e n s}^{e}\right)^{2}+\left(J_{e n c}^{e}\right)^{2}}\right\}^{2}
$$

where $J_{e n}$ can be obtained by

$$
J_{\text {ens }}=\frac{n \omega \sigma \Delta^{e}}{4 S} \sum_{i=1,2,3} A_{n c}^{i}
$$

and

$$
J_{e n c}=\frac{n \omega \sigma \Delta^{e}}{4 S} \sum_{i=1,2,3} A_{n s}^{i}
$$

where $A$ is the vector potential on each node and $S$ is the cross sectional for each material.

\section{CONCLUSION}

A numerical method called HBFEM for the analysis of the magnetic fields in switching power supplies has been proposed. This method can be effectively used to solve the harmonic problems in the magnetic field. To design and analyze switching power supplies, numerical techniques are required to determine the leakage inductance, eddy currents, and power loss. All these losses involve electromagnetic field analysis. Therefore, the numerical technique plays an important role in the analysis of switching power supplies. This paper presented a harmonic balance technique combined with the finite element method for solving the quasi-static nonlinear magnetic field problems. The magnetic field coupled with the circuits has been discussed, and a numerical model described by a system matrix equation is obtained. Several examples show the application of HBFEM to solve harmonic and eddycurrent problems. The comparison between numerical and experimental results is in good agreement.

\section{REFERENCES}

[1] F. C. Lee, "High-frequency quasiresonant converters technologies," Proc. IEEE, vol. 76, no. 4, pp. $362-376,1988$.

[2] J. G. Kassakian and M. F. Schlecht, "High-frequency high-density converters for distributed power supply systems," Proc. IEEE, vol. 76, no. 4, pp. 362-376, Apr. 1988.

[3] N. Mohan, T. M. Undeland, and W. Robbins, Power ElectronicsConverters, Applications and Design. New York: Wiley, 1989.

[4] S. A. Maas, Nonlinear Microwave Circuits. Norwood, MA: Artech House, 1988.

[5] S. Yamada and K. Bessho, "Harmonic field calculation by the combination of finite element analysis and harmonic balance method," IEEE Trans. Magn., vol. 24, no. 6, pp. 2588-2590, Nov. 1988.

[6] Y. Saito, H. Saotome, S. Yayano, and S. Yamamura, "Modeling of nonlinear inductor exhibiting hysteresis loops and its application to the single phase parallel inverters," IEEE Trans. Magn., vol. 19, no. 5, pp. 2189-2192, 1983.

[7] L. V. Kantorovich and V. I. Krylov, Approximate Method of Higher Analysis, 4th ed., translated by C. D. Benster. New York: Wiley, 1959, ch. IV.
[8] J. Lu, S. Yamada, and K. Bessho, "Development and application of harmonic balance finite element method in electromagnetic field," Int J. Appl. Electromagn. Mat., vol. 1, nos. 2-4, pp. 305-316, 1990.

[9] "Time-periodic magnetic field analysis with saturation and hysteresis characteristics by harmonic balance finite element method," IEEE Trans. Magn., vol. 26, no. 2, pp. 995-998, 1990.

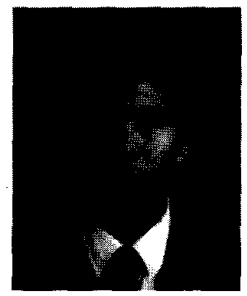

Jun Wei Lu (M'92) was born in 1955 in Shanghai, China. He graduated from the Department of Electrical Engineering, Xian Jiaotong University, China, in 1976. He received the M.Eng. degree in electrical engineering from National Toyama University, Japan, in 1988 and the Ph.D. degree in electrical and computer engineering from National Kanazawa University, Japan, in 1991.

From 1976 to 1983 , he worked with the Institute of Qinhai Electric Power Testing and Research, in China, where he has been involved in various research projects for the electrical power industry. In 1985 he began academic study and research in the area of computational electromagnetics at the Laboratory of Electrical Communications, Toyama University, Japan. Since 1988 he has worked on applied computational electromagnetics and the research and development of magnetic devices with the Laboratory of Electrical Energy Conversion, Kanazawa University. He joined the School of Microelectronic Engineering, Griffith University, in 1992, where he is now Lecturer and responsible for development of high-frequency switching power supplies for communications systems. His fields of interest include high-frequency softswitching converters, high-frequency magnetics, the application of numerical methods in power electronics and electric machines, and the computational electromagnetic and its applications in the design and analysis of RF/MW devices and circuits.

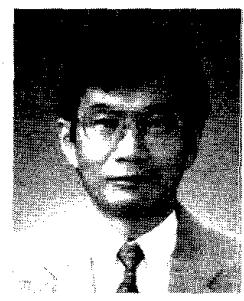

Sotoshi Yamada (M'86) was born November 22 1949, in Kanazawa, Japan. He received the B.E. and M.E. degrees from the Department of Electrical Engineering, Kanazawa University, Kanazawa, Japan, in 1972 and 1974 , respectively. He received the Dr. Eng. degree from Kyushu University, Fukuoka Japan, in 1985 .

From 1974 to 1992 , he was with the Department of Electrical and Computer Engineering, Faculty of Engineering, Kanazawa University. He has been Professor at Laboratory of Magnetic Field Control and Applications since 1992 and is engaged in research on power magnetic devices, the numerical electromagnetic field calculation, biomagnetics, etc.

Dr. Yamada is a member of the Institute of Electrical Engineers of Japan, the Magnetic Society of Japan, and Japan AEM Society.

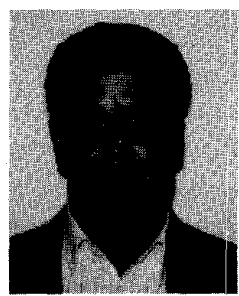

H. Barry Harrison heads the School of Microelectronic Engineering and is Chairman of the Engineering Board of Studies at Griffith University. He is also Technical Consultant to the Board of Microelectronic Technologies Pty, Ltd. (a company that was established due to his initiative) and Director of the Space Center for Microelectronic Technology $\mathrm{He}$ is also a consultant on microelectronic-related matters to other companies. He has written in excess of 200 papers, one book, and two book chapters. His research interests are in the areas of limits to scale of integration, contact modeling, and semicustom IC architecture. 